\title{
Monte Carlo Methods to Numerically Simulate Signals Reflecting the Microvascular Perfusion
}

\author{
Figueiras Edite ${ }^{1}$, Requicha Ferreira Luis F.1, \\ De Mul Frits F.M. ${ }^{2}$ and Humeau Anne 3 \\ ${ }^{1}$ Faculty of Sciences and Technology of Coimbra University, Physics Department, \\ Instrumentation Center (GEI-CI), Coimbra, \\ 2previously at University of Twente, Department of Applied Physics, Biomedical Optics \\ Group, Enschede, \\ ${ }^{3}$ Laboratoire d'Ingénierie des Systèmes Automatisés (LISA), \\ University of Angers, Angers, \\ 1Portugal \\ ${ }^{2}$ The Netherlands \\ ${ }^{3}$ France
}

\section{Introduction}

Biomedical engineering can be defined as a part of the engineering domain that aims at better understanding biological systems and proposing new tools for diagnosis and therapeutic purposes. Among the activities of the biomedical field, one is dedicated to the analysis of the very small blood vessels (microcirculation). The study of the latter is important for diagnosis and follow-up of pathologies among which we can find diabetes. This chapter deals with Monte Carlo simulations applied to the microcirculation domain.

The microcirculation comprises the blood vessels of the most peripheral part of the vascular tree. Microcirculation includes capillaries, arterioles (small arteries), venules (small veins), and arteriovenous anastomosis (shunting vessels). In what follows, only skin microcirculation will be studied. Skin microcirculation is an important and complex system for thermoregulation, skin metabolism, and transcutaneous penetration. The monitoring of skin microcirculation can be useful to assess and to better understand skin physiology and diseases.

The skin microvascular network corresponds to different compartments. Thus, the epidermis is the top layer of the skin. Epidermis is avascular. Below the epidermis, the dermal papillae contains the capillaries. The latter are responsible for the exchange of oxygen and metabolites with the surrounding tissues. Therefore, the blood perfusion through capillaries corresponds to the nutritive blood flow. The deeper dermal structures contain the arterioles, venules, and shunting vessels. These vessels feed and drain the capillary network and aim at maintaining an adequate body temperature.

In order to monitor microvascular blood flow, the laser Doppler flowmetry (LDF) technique has been proposed in the 1970s (for a review see for example Öberg, 1990; Humeau et al., 2007; Rajan et al., 2009; Cal et al., 2010). LDF allows a non-invasive and real time monitoring 
of the blood perfusion with a minimal influence in the parameters under study. It is applicable in experimental and in clinical settings.

In the LDF technique, a coherent light is brought to impinge on the tissues under study, generally through an optical fibre. The photons are scattered by the moving objects (mainly red blood cells) and by static structures. Scattering in moving objects modifies the direction and frequency of the photons according to the Doppler principle. Scattering in static structures only affects the direction of the photons. The remitted light is brought through another optical fibre to a photodetector where optical mixing of light shifted and unshifted in frequency gives rise to a stochastic photocurrent. The power spectral density of the latter depends on the number of red blood cells and their shape and velocity distribution within the scattering volume. The zero order moment scales with the concentration of moving red blood cells, provided the red blood cells concentration in tissue is low. The first moment of the photocurrent power spectrum scales with the product of the red blood cells concentration and average velocity (Bonner \& Nossal, 1981).

In most LDF devices, the light source is a laser diode having a wavelength of $780 \mathrm{~nm}$. However, other wavelengths can be used $(450-800 \mathrm{~nm})$. Within the visible range, the longer the wavelength, the deeper the transmission in tissue. In the range 600 to $1200 \mathrm{~nm}$, the light penetrates deeply into tissue because of lower scattering and absorption coefficients. Very often the light is brought to and from the tissues under study by optical fibres. In the probe tip, the fibres are generally positioned with a core centre spacing of 250 to $500 \mu \mathrm{m}$. The photons migrate in the tissue in random pathways from the transmitting to the receiving fibres. When the distance between the fibre tips increases, the average path length of the detected photons increases, as well as the measurement depth (Jakobsson \& Nilsson, 1993). Moreover, the average path length of the photons and the measurement depth depend on the optical properties of the tissue. Therefore, no comparison of perfusion values is possible between organs. That is why no absolute units are possible when using LDF.

The product of the average speed and concentration of moving blood cells in the scattering volume corresponds to the LDF signal and is generally referred as perfusion (see an example of LDF signal in Figure 1). Microvascular blood perfusion varies with time and from place to place (temporal and spatial variability). Therefore, and when using laser Doppler flowmeters based on optical fibres and thus for which the sampling volume is rather small, differences in perfusion readings appear when recording LDF signals from adjacent sites. This constitutes one of the main limitations of the LDF technique. Laser Doppler imagers have emerged providing a monitoring of the perfusion in two dimensions. LDF can be used in experimental investigations and in clinical trials. Many organs can be investigated with the LDF technique: kidney, liver, intestines, brain, skin. Moreover, the clinical applications are numerous: diabetes microangiopathy, flap monitoring, peripheral vascular disease, plastic surgery, Raynaud's phenomenon, thermal injury (see for example Ray et al., 1999; Humeau et al., 2004; Ziegler et al., 2004; Yamamoto-Suganuma \& Aso, 2009; Merz et al., 2010; Smit et al., 2010).

Due to the poor predictability of tissue perfusion for given location and time, LDF signals are rarely recorded in basal conditions. Instead, stimuli are often provoked and responses are studied to reveal possible malfunctioning of the microcirculation. All in all, the main advantages of the LDF technique are the following: non-invasiveness, continuous recordings, easy to use, strong theoretical basis. By contrast, the main drawbacks of the technique are: no absolute calibration, no possibility to distinguish between nutritive (capillary) perfusion and global tissue perfusion, no comparison possible between 


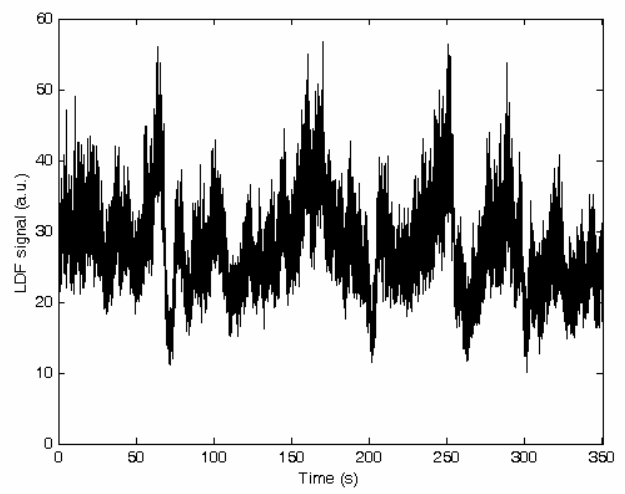

Fig. 1. Skin LDF signal recorded on the finger of a healthy subject at rest.

organs due to the variations of the photon path lengths because of the different optical properties of the tissues, variation between different individuals and in the same site in the same individual after hours, days or weeks.

In order to better understand microvascular perfusion and LDF signals, and to improve laser Doppler flowmeters, numerical simulations of LDF signals have now become necessary. Numerical simulations of LDF signals can be performed with Monte Carlo methods. The latter rely on computational algorithms using repeated random sampling to compute the simulated results.

\section{Monte Carlo simulations}

\subsection{Introduction}

In Monte-Carlo simulations, light transport in tissue is described in the form of separate photons travelling through the sample. On its way, the photon might be scattered at (or in) particles, by which the direction of the photon is changed, or the photon is absorbed. The scattering phenomenon will be determined by suitable angle-dependent scattering functions. When a boundary between two layers, or between the sample and the surrounding medium, or between an internal structure and the surrounding layer, is encountered, the photon might be reflected or refracted. This is determined by the wellknown Fresnel relations. In between these events, the photon will propagate, and the optical mean free path in that part of the sample will determine the length of the propagation path. The actual length of the contributions to the path, the angles of scattering, the choice between scattering and absorption, and between reflection and refraction, are determined by random number-based decisions.

Some extra features can be applied to the photons. For instance, photons can be thought of as scattering at particles at rest or at moving particles. This effect will cause a Doppler shift in the frequency of the photons, which can be registered. Afterwards from the Doppler shift distribution of all suitably detected photons the frequency power distribution can be derived. Several models are present for this velocity shift: unidirectional or random flow, various flow profiles and so on. Another option is to use as the light source not a beam impinging from the outside world, but a photon absorption distribution inside the sample. In this way, fluorescence or Raman scattering can be mimicked. When recording the path of 
the photons through the sample, one might deduce the path length distribution, and from that the time-of-flight distribution. The latter can be used to predict the distributions of phase delays and modulation depths encountered when performing frequency-modulation experiments.

Further, the distribution of positions where photons were absorbed can be used as the distribution of sources for calculating the photoacoustic response, to be detected using suitable detector elements (or groups of elements, to take interference effects into account) at the surface of the sample.

To start simulating the photon transport, following preparations are needed (see www.demul.net/frits):

- Definition of types of particles (optical properties, concentrations, velocities, etc.)

- Calculation of angle-dependent scattering functions for all types of particles

- Definition of the light source, either a pencil beam or a broad divergent beam or an internal source

- Definition of the sample system, consisting of one or more layers with different contents, with different optical characteristics and velocity profiles; the sample may contain "objects": (arrays of) cylinders, spheres, cones, rectangular blocks, and mirrors. See Figure 2

- Definition of the detection system, consisting of a poly-element detection window, and of its numerical aperture

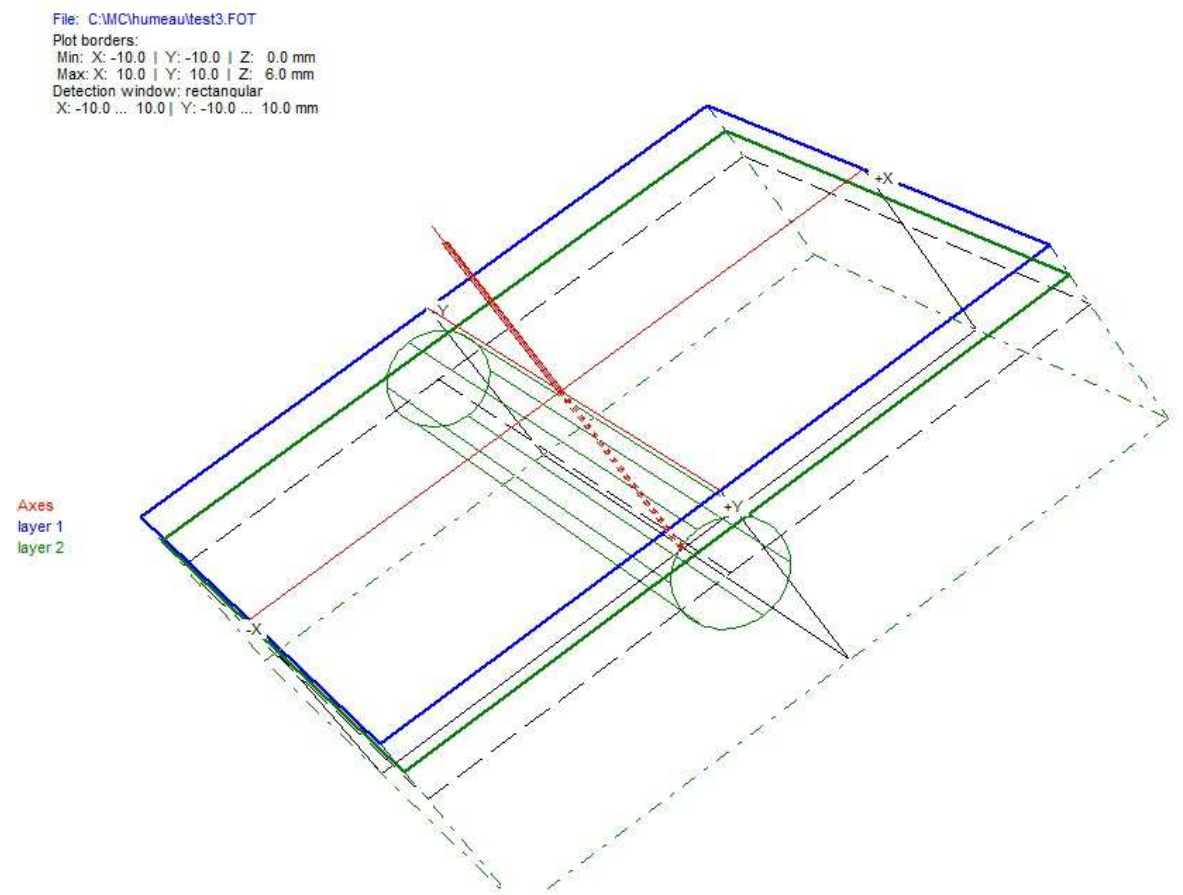

Fig. 2. Structure plot of a two-layer system with a horizontal cylindrical tube, filled with various concentrations of scattering/absorbing particles. Laser light (here pencil beam) injected along Z-axis. 
- Definition of the calculation mode: e.g. reflection or transmission, or absorption, or a combination of those

- Extra features, to follow the simulations, like LDF, photoacoustics and frequency modulation

These points will be detailed below. A computer package to carry these simulations is available (see the site www.demul.net/frits). All the processes that are presented in this section are dealt with in this computer package. The physical mathematics behind it and detailed explanations can be found in de $\operatorname{Mul}(1995,2004)$ and the site www.demul.net/frits

\subsection{Transport algorithms}

In order to describe the transport of photons through the sample, one needs algorithms for the various events that the photon may encounter. Those are: scattering or absorption, reflection or refraction at boundaries, and detection. In addition, a mechanism accounting for the destruction of irrelevant photons (e.g. photons that have travelled extremely far from the detection window) should be available.

There are two basic algorithms for handling non-zero absorption in layers or particles. Frequently the probability of absorption is taken into account as a "weight factor" for the photon. The cumulative effect of applying these subsequent factors at each scattering event will reduce its overall weight in calculating averages of relevant variables (such as intensity) over a set of emerged photons. An example is the work of Wang \& Jacques (1993). An advantage is that no photons will be lost by absorption, which can be of importance when the absorption is relatively strong.

Another algorithm does not make use of weight factors, but applies a "sudden death"method: the photon is considered to be completely absorbed at once, and will thus be removed from the calculation process. This method might be a bit more time consuming, especially when absorption is not very low in a relative sense, but it offers the advantage to study the positions where the photons actually are absorbed. In this way extra features like photoacoustics or fluorescence response can be studied. In view of this option, we have chosen for the second method (see the site www.demul.net/frits).

\subsection{Propagation}

The average translation distance $L$ for a photon in a layer or object with scattering particles of varying type, in the case of no absorption, is determined by the inverse of the effective scattering coefficient, which is the sum of the products of the concentrations and scattering cross sections of all types of particles in that layer or object. Now we can deduce the expression for the actual path length $\Delta p$ :

$$
\Delta p=L \cdot \ln (1-R),
$$

where $R$ is a random number $(0 \leq R<1)$, used for the probability $f_{s}\left(0<f_{s} \leq 1\right)$ to arrive at a path length $\Delta p$ :

$$
f_{s}=1-\exp (-\Delta p / L)
$$

However, this path might end prematurely when a boundary at an interface is met. In this case the path will partially stretch out into the medium at the other side of the interface. When dealing with this part of the path, it should be kept in mind that it has to be corrected 
in length according to the mean free path for the photons in the two media. See below for a full account.

Now the probability $f_{a}$ for absorption by the medium $l$ (layer or block) before the photon has reached the end of path $\Delta p_{\text {eff }}(\leq \Delta p)$ can be defined as:

$$
f_{a}=1-\exp \left(\mu_{a} \cdot \Delta p_{e f f}\right),
$$

where $\mu_{a}$ is the absorption coefficient.

By choosing a fresh random number, the probability for absorption on the effective path can be calculated.

Absorption in the system may have two origins, first taking place within the particles themselves, and secondly the absorption by the medium itself. Together with scattering, this leads to an "average translation length" and an "average absorption length" for the medium.

In a previous paper (Kolinko, 1997) we discussed two equivalent algorithms to determine the remaining path length after crossing an interface.

Figure 3 presents a view of a running simulation in a sample with two layers and two objects.

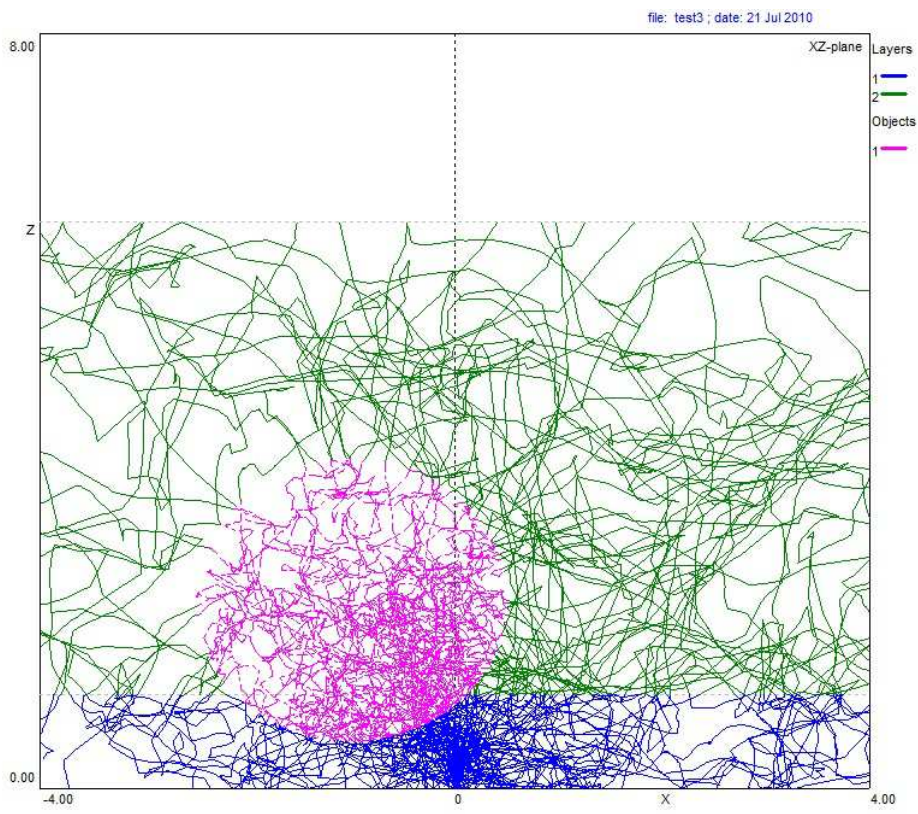

Fig. 3. Running graphics of the simulation process of the structure of Fig. 2. View in YZ-plane. Photons entering around position $(0,0,0)$. The tube (X-direction) and sphere can be seen.

\subsection{Scattering}

The probability of scattering to the direction given by the angles $\theta$ and $\varphi$ is described by the scattering function $p(\theta, \varphi)$. This function is normalized in such a way that the total scattering over the whole $4 \pi$ solid angle is unity (Figure 4 ): 


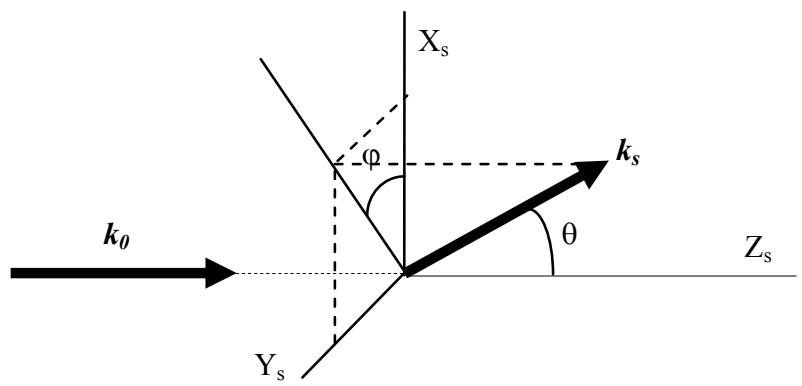

Fig. 4. Basic scattering geometry in the "scattering system" (subscript $s$ ). The incoming and scattered wavevectors are denoted by $\mathbf{k}_{0}$ and $\mathbf{k}_{\mathbf{s}}$ respectively. $|\mathbf{k}|=2 \pi / \lambda$, with $\lambda=\lambda_{\text {vacuum }} / n$ ( $n=$ refractive index of the medium $)$.

$$
\int_{0}^{2 \pi} d \varphi \int_{0}^{\pi} d \theta \cdot p(\theta, \varphi) \sin \theta=1 .
$$

For the scattering function, several models are available: Dipole- or Rayleigh-scattering, Rayleigh-Gans scattering, Mie scattering, isotropic or peaked-forward scattering. These scattering functions have been described in many textbooks. We refer here to the standard books of Van de Hulst (1957, 1981). Also models of Henyey and Greenstein (1941) and others are used (see below).

The method of determining the scattering angles $\theta$ and $\varphi$ is:

- For the azimuthal angle $\varphi: \varphi=R .2 \pi$

- For the polar angle $\theta$ a normalised cumulative function of the scattering function is used, with values between 0 and 1 , and by choosing $R$, the corresponding $\theta$ is calculated In case polarization effects have to be taken into account, the choice of the angles $\theta$ and $\varphi$ is coupled to the polarization state of the photon.

\subsection{Boundaries}

Since the program (see www.demul.net/frits) allows for insertion of special structures and objects, like tubes, spheres, mirrors and cones in the layer system, we have to deal with boundaries at flat surfaces (like those between layers) and at curved surfaces.

For flat surfaces, parallel to the layer surface (i.e. perpendicular to the Z-axis), the calculation of reflection or refraction angles is according to Snell's law. The fraction of reflected light is given by the Fresnel relations.

For curved surfaces, or flat surfaces not perpendicular to the Z-axis, the construction of local coordinate frames, along the local normal vector, is necessary, which implies foregoing and subsequent coordinate rotations from the laboratory system to the local system and back.

In de Mul (2004, see also www.demul.net/frits) the boundary expressions are derived for following curved surfaces (if applicable, with flat end surfaces at top and bottom):

- Cylinders (parallel to the layer surface, and parallel to the Z-axis, and oblique)

- Arrays of cylinders (linear or two-dimensional)

- $\quad$ Spheres, and two-dimensional arrays of spheres

- Rectangular blocks 
- Mirrors (parallel to the layer surface, and parallel to the Z-axis, and oblique)

- $\quad$ (half) toruses

Objects can stretch over layer boundaries. In all cases, carefully the path of a photon has to be followed. Is the photon reflected or refracted, how far does it propagate in the new medium, is there another object within the object, will absorption take place before a scattering event, and (in case of arrays of objects) will the photon propagate from one member of the array set to another?

\subsection{Scattering functions}

Here we will only mention the expressions for the most commonly used scattering functions. For further study, see Van de Hulst $(1957,1980)$ or the full report (de Mul, 2004, or website). Important parameters are $\mu_{s}, \mu_{s}^{\prime}$, and $\mu_{a}$, the scattering coefficient, the reduced scattering coefficient and the absorption coefficient, respectively, all expressed in $\mathrm{mm}^{-1}$, with $\mu_{s}$ and $\mu_{s}^{\prime}$ connected by $\mu_{s}^{\prime}=\mu_{s}(1-g), g$ being the averaged scattering polar angle: $g=\langle\cos \theta>$. For tissue, typical $\mu_{s}$-values are $10-200 \mathrm{~mm}^{-1}$ and with typical $g$-values of $0.90-0.99$ this leads to typical $\mu_{s}^{\prime}$ - values of $1-2 \mathrm{~mm}^{-1}$.

\section{- Dipolar (Rayleigh)}

With dipolar scattering, the particles are assumed to be so small that light scattered from different oscillating electrical dipoles in the particles will not lead to phase differences upon arrival at the point of detection (Van de Hulst, 1957, 1980). Using standard electromagnetic dipole radiation theory, or a standard Green's functions approach, we may derive for the intensities $I_{/ /}$and $I_{\perp}$, proportional to the squares of the field strengths $\left(I=1 / 2 c \varepsilon_{m} E^{2}\right)$, thus:

$$
\begin{aligned}
& I_{/ /}=\frac{\alpha^{2} k^{4}}{\left(4 \pi \varepsilon_{m}\right)^{2} r^{2}} \cos ^{2} \theta \cdot \cos ^{2} \varphi \cdot I_{0} \\
& I_{\perp}=\frac{\alpha^{2} k^{4}}{\left(4 \pi \varepsilon_{m}\right)^{2} r^{2}} \sin ^{2} \varphi \cdot I_{0} .
\end{aligned}
$$

For natural light the total intensity is given by:

$$
I_{\text {nat }}(\theta)=\frac{\alpha^{2} k^{4} I_{0}}{\left(4 \pi \varepsilon_{m}\right)^{2} r^{2}} \frac{1+\cos ^{2} \theta}{2} .
$$

\section{- Rayleigh-Gans}

When particles grow larger, the phase differences of scattered waves arriving at the detection point from different source points in the scattering medium, cannot be neglected any more. For small differences in the dielectric constant between particles and surrounding medium, the intensity I will be proportional to:

$$
I \sim \frac{1+\cos ^{2} \theta}{2} k^{4} V^{2} \frac{(m-1)^{2}}{(2 \pi)^{2}}|R(\theta, \varphi)|^{2},
$$

with:

$$
R(\theta, \varphi)=\frac{1}{V} \iiint \exp (i \delta) \cdot d V
$$


The phase-difference $\delta$ is given by $\boldsymbol{k} \bullet\left(r-r_{O}\right)$, where $\boldsymbol{r}$ and $\boldsymbol{r}_{O}$ are the position vectors from the scattering volume element under consideration and the origin in the sample.

\section{- Mie}

In principle, the rigorous scattering theory, as developed by Mie (see ref. in Van de Hulst, 1957, 1981), presents analytical expressions for all kind of particles. It departs from the Maxwell equations and solves the scalar part of the wave equation, taking boundary conditions into account. This leads to complicated expressions for the components of Van de Hulst's scattering matrix, which are only tractable when treated numerically. See Figure 5 for an example.

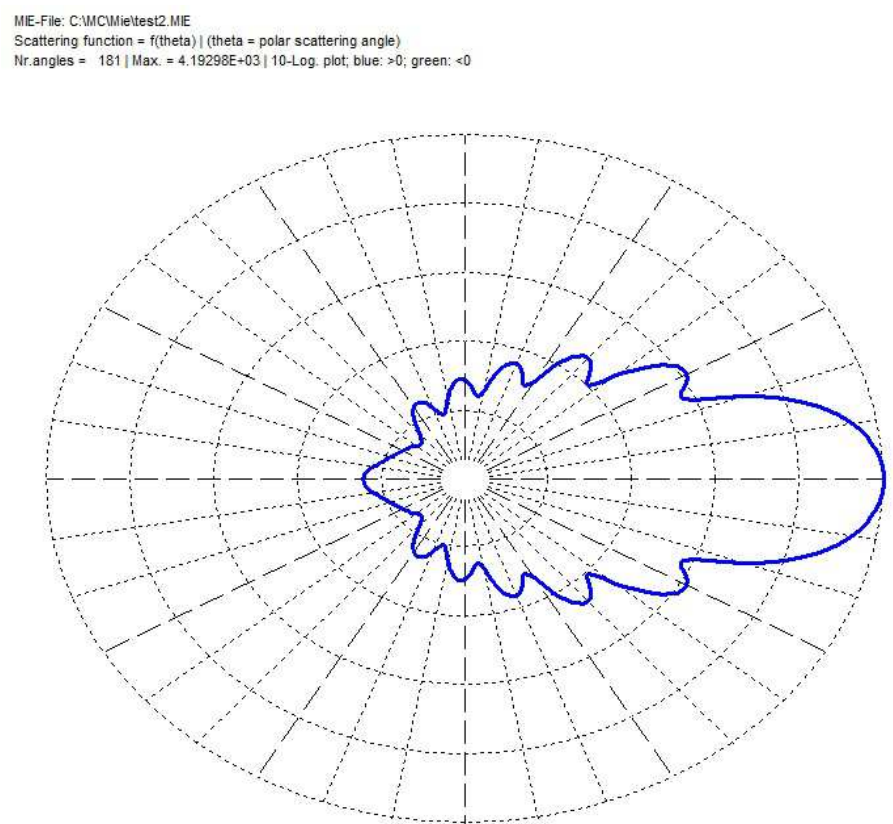

Fig. 5. Example of a MIE-file. Scattering function according to the Mie-formalism (weight 1.0) + Henyey-Greenstein with $g=0.80$ (weight 0.1 ).

\section{- Henyey-Greenstein}

The scattering function of Henyey \& Greenstein (1941) originates from the astronomical field, to calculate the scattering by cosmic particle clouds. Since it can be written in a closed analytical form, it can be used as a fast replacement for the Mie-functions. The function reads:

$$
p_{H G}(\theta, \varphi)=\frac{1}{4 \pi} \frac{1-g^{2}}{\left(1+g^{2}-2 g \cdot \cos \theta\right)^{3 / 2}},
$$

where $g$ is the averaged cosine of the polar angle $\theta$ of the scattering events. This function is normalised to unity upon integration over $4 \pi$ solid angle. It only describes the angledependent behaviour of the scattering. The calculation of the scattering cross section has to be done by other means. One option is to insert the total scattering cross section as obtained 
by Mie-scattering (or another approach, if applicable) as a separate factor in the HenyeyGreenstein expression.

\section{- Other functions}

Other scattering functions are: isotropic scattering, peaked forward, or Gegenbauer (which is an extension of a Henyey-Greenstein-function). We will not deal with those here.

\subsection{Light sources}

For the injection of photons, one can imagine various mechanisms. Most general is the pencil beam, entering from the top. However, other beam profiles can be used as well. In de Mul (2004, see web site) several options are implemented: pencil beams (perpendicular and oblique), divergent beams, broad parallel beams, ring-shaped beams, isotropic injection and internal point sources (one point or distributed).

Distributed internal sources can be used in simulating Raman or fluorescence scattering, consisting of (1) a simulation of absorptions, and (2) injection of new photons from the positions of absorption.

\subsection{Detection}

We may distinguish between external detection (at the top or bottom of the sample system: "reflection" or "transmission", or at an internal layer or object boundary) or internal detection (upon an absorption event). In this way, the scattering inside a sphere (a human head?) can be detected.

\subsection{Photon path tracking}

The tracking of the path of the photon, i.e. recording the coordinates of the scattering events and of the intersections with interfaces, can easily result in enormous files. With a scattering coefficient $\mu_{s}$ of about $10-20 \mathrm{~mm}^{-1}$ and a $g$-factor (average of the cosines of the polar scattering angles) of about $0.80-0.90$, in each $\mathrm{mm}$ of the path about $1 / \mu_{\mathrm{s}} \approx 10$ scattering events will take place. However, due to the large $g$-factor, the scattering will be predominantly in forward direction and it will only be after about $1 / \mu_{s}^{\prime} \approx 1 \mathrm{~mm}$ that the direction of the photon can be considered as randomised. Therefore, in those cases it is better to register only part of the events, namely those at intervals of $1 / \mu_{s}^{\prime}=1 \mathrm{~mm}$, which will decrease the storage space to 144 Mbytes per simulation.

Therefore, the program offers the options of recording the paths at intervals of $1 / \mu_{\mathrm{s}}$ or $1 / \mu_{s}^{\prime}$ (see www.demul.net/frits).

Photons originating from a pencil beam and emerging at equal distances $d$ from the point of injection but at different positions on that ring are equivalent. However, visualisation of those tracks will end up in an un-untwinable bunch. Therefore, to clarify viewing we may rotate the whole paths around the axis of the pencil beam to such an orientation as if the photons all emerged at the same position on the ring, e.g. the crossing point with the X-axis. See Figure 6 for an example of the path tracking method.

\subsection{Special Features: laser Doppler flowmetry}

Some special features are incorporated in the program (available at www.demul.net/frits). LDF is the oldest feature, built in from the beginning of the development of the program, and meant to support measurements of laser Doppler perfusion flowmetry in tissue. 


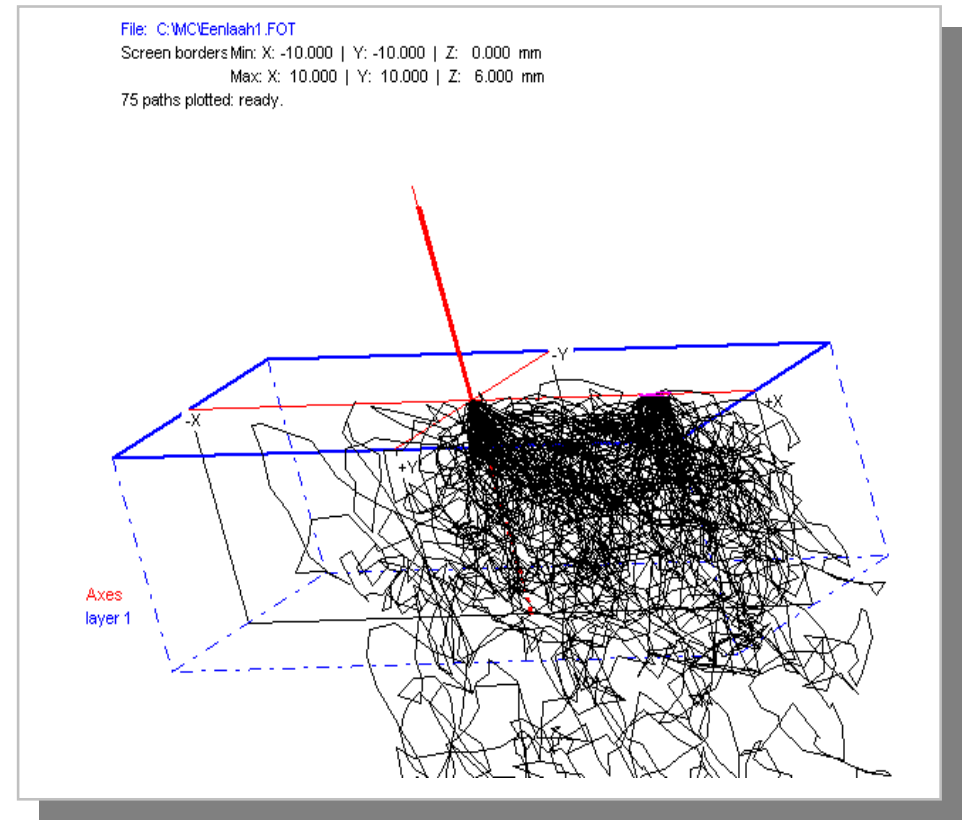

Fig. 6. Photon path tracking: photon "bananas" arising by scattering from beam entrance point to exit area (between 5 and $6 \mathrm{~mm}$ ). For clarity, all photon paths were rotated afterwards as if the photons had emerged on the $+X$-axis.

Photoacoustics has been added to simulate the acoustic response to pulsed light. Frequency modulation is a modality adding extra information using path length-dependent phase delay information. Here we only deal shortly with LDF.

As mentioned previously, LDF makes use of the Doppler effect encountered with scattering of photons in particles when those particles are moving. The principles are shown in Figure 7. Using the definitions of the variables given in that Figure, the Doppler frequency is given by:

$$
\omega_{D}=\left(\mathbf{k}_{s}-\mathbf{k}_{0}\right) \bullet \mathbf{v},
$$

and with:

$$
|\delta \mathbf{k}|=2 k \cdot \sin \frac{1}{2} \theta
$$

we find:

$$
f_{D}=\frac{k v}{\pi} \sin \frac{1}{2} \theta \cdot \cos \alpha
$$

When applied to tissue, frequently the angles $\theta$ and $\alpha$ might be considered randomised. This is due to three reasons:

- Preceding scattering by non-moving particles might cause the direction of the photons to be randomised upon encountering moving particles 


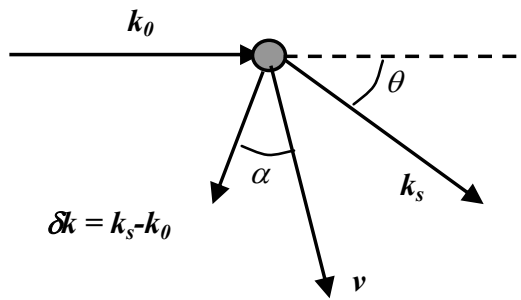

Fig. 7. Principles of LDF. The particle has a velocity $\mathbf{v}$. Vectors $\mathbf{k}_{\mathbf{0}}$ and $\mathbf{k}_{\mathrm{s}}$ denote the incoming and scattered light wave vectors, and $\delta \mathbf{k}$ is the difference vector.

- Most important moving particles are blood cells in capillaries. Due to the (more or less) random orientation of the capillaries the velocities will have random directions

- Travelling from injection point to detection point, in general the photons will encounter many Doppler scattering effects, with random velocities and orientations

All three effects will broaden the Doppler frequency distribution, which ideally would consist of one single peak, to a smooth distribution as in Figure 8. This means that it is not possible to measure the local velocity, but we only may extract information about the averaged velocity over the measuring volume. The averaging concerns the three effects mentioned above.

There are two options to record these LDF-spectra: homodyne and heterodyne, depending on the relative amount of non-shifted light impinging on the detector. The first is the mutual electronic mixing of the Doppler-shifted signals, and the second is the mixing of those signals including mixing with non-shifted light, which can be overwhelmingly present. The resulting frequency and power spectra (which is the autocorrelation function of the frequency spectrum) will look as sketched in Figure 8.
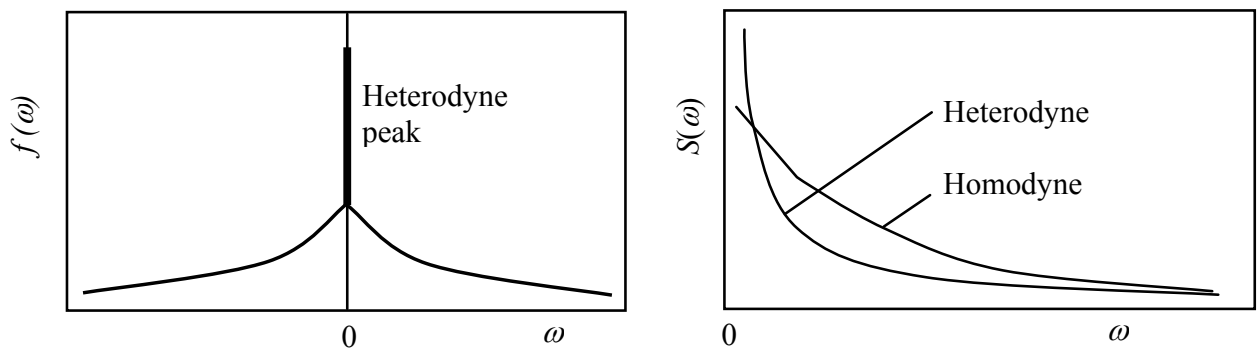

Fig. 8. Homodyne and heterodyne frequency spectra $f(\omega)$ and power spectra $S(\omega)$. Normally the heterodyne peak is much higher than the signals at non-zero frequencies.

To characterize the frequency spectra use is made of moments of the power spectrum $S(\omega)$, defined as:

$$
M_{n}=\int_{0}^{\infty} \omega^{n} \cdot S(\omega) \cdot d \omega
$$

and the reduced moments: 


$$
M_{n}{ }^{\prime}=\frac{M_{n}}{M_{0}{ }^{n}} .
$$

The zeroth moment is the area under the power spectrum itself, and can be considered as proportional to the concentration of moving particles in the measuring volume. Bonner \& Nossal (1981) showed that the first moment $M_{1}$ is proportional to the averaged flow, while the ratio of the reduced moment $M_{1}^{\prime}=M_{1} / M_{0}$ will be proportional to the averaged velocity. Analogously, the reduced moment $M_{2}{ }^{\prime}=M_{2} / M_{0}{ }^{2}$ will be proportional to the average of the velocity-squared.

\section{Monte Carlo simulations applied to the microcirculation domain}

Monte Carlo simulations have been used since the early nineties to provide a better understanding on LDF measurements. At the beginning, LDF signals were simulated using very simple single layer flow models. Later, complex physical models constructed with the purpose to validate and calibrate laser Doppler flowmeters were proposed. LDF signals were generally simulated in models mimicking the skin and then compared with real data recorded in skin.

Monte Carlo simulations allow studying the influence of tissue parameters (as optical properties and blood velocities) as well as probe configurations and laser light wavelengths on LDF signals. Monte Carlo simulations have already provided information 1) on the blood microcirculatory flow depth measurements in skin (non-invasive measurements) or in other organs (invasive measurements), 2) on the determination of the photon path length, 3) on the dependence of LDF signal on multiple scattering, and 4) on the prediction of the speed distributions of moving particles.

In the LDF domain, Monte Carlo simulations were used, for the first time, by Jentink et al. (1990). It was the first time that the frequency shifts, due to the moving particles, were included in light propagation simulations in scattering and absorbing media. Monte Carlo simulations were used to study the influence of the optical probe configuration and the multiple scattering of photons by moving particles in living tissues. A very simple flow model, consisting of a homogeneous slab with spheres acting as scatterers, has been used. The spheres were moving in random directions, which permitted the simulation of different velocity distributions. The velocity angular distribution was modelled with Mie formula. Light absorption was taken into account by including a constant probability with two terms, one due to the absorption by the spheres, and a smaller one which represented the absorption between spheres. Ring shaped detectors were attached in a concentric arrangement around the light beam and heterodyne detection was assumed. Homodyne detection was ignored. It was observed that, with the increase of source-detector distance, the sampling volume increased whereas the intensity of the signal decreased (Jentink et al., 1990). Therefore, the authors suggested the use of different source-detector separations in order to differentiate perfusion in different skin layers. The limited computational speed available in the early nineties was a serious limitation for the first studies using Monte Carlo simulations.

A more complex skin tissue model, consisting of three different homogeneous layers (epidermis with $0.1 \mathrm{~mm}$ thickness, dermis- 1 with $0.2 \mathrm{~mm}$ thickness, and dermis-2 with $200 \mathrm{~mm}$ thickness) was used in Monte Carlo simulations by Koelink et al. (1994). The goal was to determine the sampling volume in LDF measurements using two different 
wavelengths, $633 \mathrm{~nm}$ and $800 \mathrm{~nm}$, and two different source-detector separations, 0.1 and $2 \mathrm{~mm}$. The optical parameters used were 0.015 and $0.01 \mathrm{~mm}^{-1}$ for the absorption coefficients for 633 and $800 \mathrm{~nm}$, respectively, for all three layers. The scattering coefficients used were 25 and $15 \mathrm{~mm}^{-1}$ for epidermis for 633 and $800 \mathrm{~nm}$, respectively. For dermis-1 and dermis-2, they were of 11.2 and $6.8 \mathrm{~mm}^{-1}$ for 633 and $800 \mathrm{~nm}$, respectively. The refractive indexes were 1.5 for epidermis and 1.4 for dermis- 1 and for dermis-2. A Henyey-Greenstein phase function with an anisotropy factor $g$ equal to 0.85 for static tissue was used. Microcirculation was assumed in the two deeper layers with a concentration of $1 \times 10^{4}$ red blood cells $/ \mathrm{mm}^{3}$ in both layers, in random directions. A velocity equal to $1 \mathrm{~mm} / \mathrm{s}$ was simulated in dermis-1, whereas for dermis-2 the velocity was equal to 1 or $4 \mathrm{~mm} / \mathrm{s}$ in different simulations. The scattering cross section, $\sigma_{s}$, of the red blood cells used was equal to 25.4 and $15 \mu \mathrm{m}^{2}$ for 633 and $800 \mathrm{~nm}$, respectively. The absorption cross section, $\sigma_{a}$, was equal to 0.065 and $0.042 \mu \mathrm{m}^{2}$ for 633 and $800 \mathrm{~nm}$, respectively. For the scattering phase function of red blood cells the Rayleigh-Gans phase function was applied with an anisotropy factor $g$ equal to 0.985 and 0.98 for 633 and $800 \mathrm{~nm}$, respectively (Koelink et al., 1994). The aim of this work was to distinguish the superficial from the deeper blood vessels microcirculation. Measurements in skin were compared with simulations showing a reasonable agreement (Koelink et al., 1994). With the purpose to compare real data and Monte Carlo simulations of flow, de Mul et al. (1995) introduced phantoms (physical flow models) in Monte Carlo simulations. The Monte Carlo algorithm used is explained in Section 2. Unlike biological tissues, a phantom permits the control on LDF measurements of relevant parameters, such as scatterers velocity and concentration, optical properties, and so on. Two models were studied: a liquid flow model consisting of a set of liquid layers, and a solid model based on gelatine layers, both with a concentration of 1.25 to $1.9 \times 10^{6} \mathrm{~mm}^{-3}$ of polystyrene spheres acting as scatterers. The goal was also to investigate the relationship between source-detector separation and the photons sampling depth, for different incident angles of the laser beam. The angular scattering distribution for the polystyrene spheres was given by the Mie formulas with an anisotropy factor $g=0.91$. The scattering and absorption cross section of the spheres were set to 5.5 and $0.03 \mu \mathrm{m}^{2}$, respectively, at $780 \mathrm{~nm}$. The effects of homodyne and heterodyne scattering were also investigated. The comparisons showed reasonable to good agreement between simulations and real measurements using the phantom (de Mul et al., 1995).

A more complex fluid model applied to Monte Carlo simulations was presented by Steenbergen \& de Mul (1997). In order to study the phantom capability to mimic real tissue, the model had optical properties and layered structure similar to those of living tissues. The Monte Carlo algorithm used is explained in Section 2. This phantom, consisting of scattering and absorbing films separated by matching oil, was used to evaluate the consequences of the stratified structure of the phantom in comparison with real tissues, that have no mismatching layers. The phantom was modelled as a semi-infinite repetitive laminate of scattering and absorbing layers with $0.08 \mathrm{~mm}$ thickness separated by a transparent colourless layer of resin or oil with $0.005 \mathrm{~mm}$. The laser light source entered the phantom as a pencil beam and the scattered light was detected in a concentric region (radius equal to $2.5 \mathrm{~mm}$ ) with the pencil beam. Concerning the optical properties, the angular scattering function used was the Henyey-Greenstein distribution with an anisotropy factor $g=0.9$. The scattering coefficient used was $\mu_{s}=5,10,20$ and $40 \mathrm{~mm}^{-1}$ which gave a reduced scattering coefficient equal to $\mu_{s}^{\prime}=0.5,1,2$ and $4 \mathrm{~mm}^{-1}$ respectively. The absorption coefficient used was $\mu_{a}=0.005 \mathrm{~mm}^{-1}$. The refractive index of the phantom matrix was 1.52 . The algorithm used was similar to the one developed by de Mul et al. (1995). When the photon travelled 
between a transparent layer and a scattering layer, a new path length was determined using a random generator (the path length which has been interrupted by the medium boundary was disregarded). At the end, the mismatching problems revealed not to being as restrictive as it might have been expected (Steenbergen \& de Mul, 1997).

The coherence effects in the detection of Doppler signals have also been investigated with Monte Carlo simulations. Measurements in gelatin phantoms with polystyrene scatterers was built to mimic skin tissue characteristics and for calibration and standardization of perfusion tools (de Mul et al., 1999). Homodyne and heterodyne detection were investigated. For the homodyne experiments, a phantom consisting of just one moving layer with $11 \mathrm{~mm}$ thickness was used. For the heterodyne experiments, the phantom consisted of one static and one moving layer with 4 and $11 \mathrm{~mm}$ thickness, respectively, with the same scatterers concentration. The simulation model consisted of five layers: an air gap between probe and first layer, a fixed layer, another air gap between the first and second layer, a moving layer, and an absorbing layer. The homodyne simulations did not match the corresponding measurements quite properly, showing a too broad Doppler power spectrum that was, in part, reduced by the implemented coherence correction (de Mul et al., 1999).

The influence of the optical properties and the source-detector separation on the sampling depth in LDF measurements were also studied using a sophisticated tissue-like phantom (Larsson et al., 2002). Monte Carlo simulations mimicking the phantom were used to validate the measurements. The tissue phantom and the simulation model consisted of a set of parallel static layers ( $95 \mu \mathrm{m}$ thickness) with different optical properties, separated by rotatable moving layers ( 20 to $22 \mu \mathrm{m}$ thickness). A laser source of $632.8 \mathrm{~nm}$ was used and the backscattered light was guided to the detector by 7 fibres, with a numerical aperture of 0.37 and a diameter of $230 \mu \mathrm{m}$, arranged in a row. Hollow polystyrene microspheres, with a diameter of $1 \mu \mathrm{m}$, were used as scatterers, and optical absorbers were added to the static layers. The phantom had 4 windows, where the probe could be placed, each one with different combinations of optical properties (14.66 and $0.212 \mathrm{~mm}^{-1}, 44.85$ and $0.226 \mathrm{~mm}^{-1}$, 14.8 and $0.0405 \mathrm{~mm}^{-1}$ and 45.55 and $0.0532 \mathrm{~mm}^{-1}$ for $\mu_{a}$ and $\mu_{s}$, respectively). The probability distribution of the photon scattering angle was modelled using the Henyey-Greenstein phase function with an anisotropy factor $g=0.815$. Two models were simulated: 1 ) with one single rotating disk having 14.66 and $0.212 \mathrm{~mm}^{-1}$ for $\mu_{a}$ and $\mu_{s}$, respectively, for two different velocities ( 0.7 and $2.2 \mathrm{~mm} / \mathrm{s}$ ); and 2) with the same optical properties as the four windows described above, where single moving disks and multiple moving disks at $1 \mathrm{~mm} / \mathrm{s}$ could be modelled. Measured and simulated data showed good correlation and the simulations showed that the sampling depth decreases with the increase of $\mu_{a}$ or $\mu_{\mathrm{s}}$ and it increases with the increase of source-detector fibre separation (Larsson et al., 2002).

Other Monte Carlo simulations were used to predict sampling depth of light scattering in skin, in different situations. The sampling depth for a laser Doppler perfusion imaging system, using a simplified model with a single moving layer, was also analyzed (Rajan et al., 2008).

Other authors investigated the use of a high power laser source $(20 \mathrm{~mW})$, with $785 \mathrm{~nm}$ laser light source and a source-detector distance of $4 \mathrm{~mm}$ (Clough et al., 2009).

Based on Monte Carlo simulations of light propagation, the measurement depth/volume was also estimated for various tissues models (muscle, liver, gray matter, white matter, and skin). Both probe-based and imaging systems were studied, at the wavelengths of $453 \mathrm{~nm}$, $633 \mathrm{~nm}$ and $780 \mathrm{~nm}$ (Fredriksson et al., 2009). Thus, using Monte Carlo simulations, typical measurement depths and volumes for simulated perfusion have been presented, for various 
types of biological tissues and system setups. The simulations were not compared with in vivo measurements (Fredriksson et al., 2009).

A skin model, that can be used to estimate skin-like tissue perfusion in absolute units, was also presented (Fredriksson et al., 2008). The impact on LDF measurements of parameters such as layers thickness, blood concentrations, melanin concentration in epidermis, $\mu_{a}, \mu_{s}$ and $g$ for blood and skin layers were evaluated with Monte Carlo simulations. The simulated spectra generated for 7000 different skin configurations, for two different sourcedetector separations $(0.25$ and $1.2 \mathrm{~mm})$, and a $780 \mathrm{~nm}$ laser light source, were compared with in vivo data. The goal was to validate the best fit model with the measured spectra. In this study, the skin model had 6 layers (epidermis, papillary dermis, superior blood net, reticular dermis, inferior blood net, and subcutis). Moreover, different blood concentrations were chosen with three representative velocities $(0.3,3.0$, and $30 \mathrm{~mm} / \mathrm{s})$ in each layer, and with a parabolic profile (between 0 and twice the mean velocity of the scattering blood component). The simulated source and detector were configured to mimic the probe used. The calculated spectra were compared with measurements carried out on the forearm and on the finger pulp skin, without heating, and with heat provocations on the forearm skin. At each scattering occasion it was decided, based on the concentration of blood, the scattering coefficient of the blood and the static matrix, if the photon was to be scattered by the static matrix, or by a moving red blood cell, causing Doppler shift. The velocity component $(0.3,3$, or $30 \mathrm{~mm} / \mathrm{s}$ ) that produced the Doppler shift, in simulations, was randomly chosen based on the scatterers concentration. The optical power spectrum for the two source-detector separations was calculated. The distribution of accumulated frequency shifts for the detected photons and the Doppler power spectrum was also calculated as the autocorrelation of the optical power spectrum. For the photon launch, a variance reduction method, the implicitly capture, was used. In this method, after the first scattering, the photon was splitted into 50 new photons, each one with 1/50 of the weight of the original photon and it was scattered in random directions. Then, in the following successive Doppler scattering occasions, the photon was splitted again into two new photons, if the total frequency shift of the photon exceeded $n \times 10 / 6 \mathrm{kHz}(n=1,2, \ldots, 6)$. One photon could thus be splitted into, at most, $50 \times 2^{6}=3200$ photons. A good combination of thickness of the model and of blood concentrations that produced simulated Doppler power spectra that agreed well with measured Doppler power spectra, was found (Fredriksson et al., 2008).

Methods for photon path length determination in LDF have also been proposed by Monte Carlo simulations (Jakobsson \& Nilsson, 1993; Nilsson et al., 2002; Larsson et al., 2003; Varghese et al., 2007). Thus, Jakobsson \& Nilsson (1993) used one-layer models of skin, liver and brain tissues to study path length distributions for different probe geometries at a wavelength of $633 \mathrm{~nm}$. The 3D pathways of single photons were computed and stored. Information as the penetration depth, sampling depth, and total photon path length between source and detector were stored. The Henyey-Greenstein phase function was used as the density function of the scattering angle. The tissues optical properties $\mu_{a}, \mu_{s}$ and $g$ (in $\mathrm{cm}^{-1}$ for $\mu_{a}$ and $\mu_{s}$ ) were respectively set equal to 2,188 and 0.8 for skin, $2.3,313$ and 0.68 for liver, 1.3, 48.8 and 0.96 for brain. For the whole blood (unmodified blood), the values were respectively equal to 18,320 and 0.99 . For each distance travelled by a photon, the probability of absorption was calculated. If absorption occurred, the photon pathway was terminated. Different perfusion profiles were simulated in skin tissues model and the optical parameters $\left(\mu_{a}, \mu_{s}, g\right)$ were changed stepwise, one by one. The influence of different red blood cell concentrations, in skin tissue was also simulated. 
Using a one-layer model with a wide range of optical properties, relevant to human skin, the average path length for various source detector separations up to $2 \mathrm{~mm}$ was simulated, using Monte Carlo methods (Nilsson et al., 2002). The Monte Carlo simulation software used was developed by de Mul (de Mul et al., 1995). A reference space model built to develop path length estimation methods with 144 different sets of optical properties $\left(\mu_{a}, \mu_{s}\right.$, and $g$ ) and a validation space model for evaluation of the accuracy of the path length estimations methods with 75 different sets of optical properties were defined. Different methods for predicting the path length were investigated. A multiple polynomial regression method, based on spatially resolved diffuse reflectance, proved to be the most effective in predicting the average path length as a function of source-detector separation (Nilsson et al., 2002).

Larsson et al. (2003) estimated the photon path length and optical properties for source detector separation up to $1.61 \mathrm{~mm}$. They used a simulated model consisting of a semiinfinite slab, with $100 \mathrm{~mm}$ thickness, with a low concentration of moving scatterers and with a constant velocity ( $v=1 \mathrm{~mm} / \mathrm{s}$ ). de Mul software (de Mul et al., 1995) was used and the scattering events were simulated with the modified Henyey-Greenstein phase function for a $632.8 \mathrm{~nm}$ laser source. Four different sets of optical properties were simulated. Measurements, in vivo, at different human skin sites and, in vitro, in two phantoms were used together with simulations in order to obtain the estimated $\mu_{a}$, the estimated reduced scattering coefficient $\mu_{s}^{\prime}$, estimated photon path length and normalized and linearized LDF perfusion. The path length estimations were applied for normalization of the estimated perfusion, removing its optical properties dependency (Larsson et al., 2003).

The optical path lengths of shifted and unshifted light, as well as the path length dependent Doppler broadening were measured in a two-layer tissue phantom (with a superficial static layer of different thickness) with a phase modulated low coherence Mach-Zehnder interferometer (Varghese et al., 2007). Validation of the experimentally determined thickness of the static layer and the optical path length distributions were done with the Doppler Monte Carlo methods. The simulated phantom consisted of a static scattering layer with variable thickness (between 0.1 and $0.9 \mathrm{~mm}$ ), between two static glass layers with $0.15 \mathrm{~mm}$, a dynamic layer with $20 \mathrm{~mm}$, and a fifth layer with high absorption characteristics $\left(\mu_{a}=\right.$ $10 \mathrm{~mm}^{-1}$ ). The static and dynamic scattering layers had the same optical properties as they had the same scatterers - polystyrene sphere suspension. The refractive index was $1.33, \mu_{a}$ and $\mu_{s}^{\prime}$ were set to 0.001 and $2 \mathrm{~mm}^{-1}$, respectively, and $g=0.85$ for Henyey-Greenstein scattering phase function. The thickness of the static layer was estimated from the minimum optical path length of Doppler-shifted light. A good agreement between experimentally determined thickness of the static layer and Monte Carlo simulation was obtained (Varghese et al., 2007). The method was able to measure path length resolved information of nonshifted and shifted Doppler fractions of photons (Varghese et al., 2007).

Using Monte Carlo simulations, a few authors worked on the prediction of the speed distributions of moving particles based on the laser Doppler spectrum decomposition (Fredriksson et al., 2006; Larsson \& Stromberg, 2006; Liebert et al., 2006). As the velocity depends on the dimension of the blood vessels, the prediction of the speed distributions could lead, in vivo, to differentiate between capillary and arterial blood flow. Various uniform and Gaussian speed distributions of particles, moving in the turbid media, were simulated in order to study the relation between the calculated speed distributions of moving particles and the simulated distribution using Monte Carlo simulations (Liebert et al., 2006). The Henyey-Greenstein phase function was used for the calculations and several 
anisotropy factors of the medium were simulated (Liebert et al., 2006). The optical properties, $\mu_{a}, \mu_{s}^{\prime}$ and refractive index $n$ were set to $0.01 \mathrm{~mm}^{-1}, 1 \mathrm{~mm}^{-1}$ and 1.4 , respectively. A laser light source of $780 \mathrm{~nm}$ was used. The backscattered photons were collected in concentric ringshaped detector at $1 \mathrm{~mm}$ from the source and a concentration of $1 \%$ of moving scatterers was used for simulations. The theoretical background of single and multiscattering were presented, but it was validated by Monte Carlo simulations for singlescattering, only. The calculated speed distribution of moving particles matched with the assumed simulated Gaussian distributions of the moving particles speeds (Liebert et al., 2006).

An algorithm for velocity resolved perfusion measurements, that characterizes the microvascular blood flow in three different velocities, was suggested by Larsson \& Strömberg (2006). This algorithm was derived by fitting a set of predefined Monte Carlo simulated, single velocity spectra, to a measured, multiple velocity LDF spectrum, based on single Doppler scattering event. The proposed method yields three concentration measurements, each associated with a predefined, physiologically relevant, absolute velocity. A perfusion phantom with a microsphere solution (or diluted blood), and with single or double-tube flow was used for validation. A parabolic flow profile, with average flow velocity equal to the real flow velocity, was simulated using the two phantoms with different experimental setups. Mie theory was used for the microspheres scattering angle distribution and Gegenbauer kernel phase function was used for blood. This study showed that the LDF signal can be separated into, at least, three different velocity regions (Larsson \& Strömberg, 2006).

Another method based on Doppler power spectrum decomposition into a number of flow velocity components, measured in absolute units $(\mathrm{mm} / \mathrm{s})$, was proposed (Fredriksson et al., 2006). With Monte Carlo simulations, the number of Doppler shifts, and the total Doppler shift, were recorded for each detected photon. From the simulated optical Doppler spectrum resulting from the detected photons, the Doppler power spectrum was calculated. The shift distributions thus obtained were used for measured, and calculated, spectra comparisons. With the simulated shift distribution, a Doppler power spectrum was calculated, originating from a certain combination of velocity components using a mathematical model. The non linear Levenberg-Marquardt optimization method was used to fit the calculated and the measured Doppler power spectra, giving the set of velocity components in the measured sample. Evaluation of the method was achieved with a multi-tube flow phantom, perfused with polystyrene microspheres infusion, or human blood. The results showed good velocity components estimations for low velocities and low concentrations of moving scatterers, but the opposite was found for high velocities and high concentrations. The reason for that was attributed to the physical characteristics of the phantom used (Fredriksson et al., 2006).

A recent study (Binzoni et al., 2009) suggested a reinterpretation of the Monte Carlo simulation in order to obtain more information, relevant to LDF measurements. The authors suggested a method for photo-electric current determination in Monte Carlo simulations which will allow that any algorithm used in real LDF instrument could be tested and validated.

Our research group is using Monte Carlo simulations in order to validate a new laser Doppler flowmeter prototype for depth flow discrimination in skin. This prototype uses different wavelengths $(635,785$, and $830 \mathrm{~nm})$ and different source-detector fibre distances $(0$, $0.14,0.25$, and $1.2 \mathrm{~mm}$ ). The prototype is evaluated, in vitro, in a phantom consisting of six layers of Teflon ${ }^{\circledR}$ microtubes (with 0.3 and $0.76 \mathrm{~mm}$ inner and outer diameter, respectively). 
Skimmed milk is used as the moving fluid (Figueiras et al., 2010). Milk has been chosen because it has various components that act as scatterers (carbohydrates, fat, and protein). Moreover, it does not sediment like microspheres, and it has similar behaviour to intralipid solutions (Waterworth et al., 1995). Finally, milk is easier for handling than blood and, besides, it is cheaper. However, as milk is unstable, we use the same solution of milk for one day only. Milk is pumped in the microtubes with a motorized syringe with different velocities: 1.56, 3.12, 4.68, 6.25, 7.78, and $9.35 \mathrm{~mm} / \mathrm{s}$. The prototype will also be evaluated, in vivo, in healthy human subjects. Real measurements and simulations will then be compared. For the three wavelengths and for the four different source-detector fibre separations, LDF simulations are carried out with a skin model similar to the one proposed by Fredriksson et al. (2009), and with the phantom. For the skin layers, $\mu_{a}$ is set equal to $0.15,0.1$, and $0.0122 \mathrm{~mm}^{-1}$, for 635,785 , and $830 \mathrm{~nm}$, respectively. $\mu_{s}$ is set equal to 20,13 , and $18 \mathrm{~mm}^{-1}$ for 635,785 , and $830 \mathrm{~nm}$, respectively. Regarding the blood optical properties, $\mu_{a}$ is set equal to $0.34,0.5$, and $0.52 \mathrm{~mm}^{-1}$, for 635,785 , and $830 \mathrm{~nm}$, respectively, and $\mu_{\mathrm{s}}$ is set equal to 16,13 , and $11 \mathrm{~mm}^{-1}$ for 635,785 , and $830 \mathrm{~nm}$, respectively. Concerning the phantom simulations with a $635 \mathrm{~nm}$ laser source, the refractive index for milk is 1.346, $\mu_{a}$ and $\mu_{s}$ are 0.00052 and $52 \mathrm{~mm}^{-1}$, respectively. For the tubes, the refractive index is $1.367, \mu_{a}$ and $\mu_{s}$ are equal to 0.001 and $167 \mathrm{~mm}^{-1}$, respectively. The software used was developed by de Mul (1995). The mean photon Doppler shifted depths obtained for the modelled skin and for the phantom are presented in Figure 9. For skin simulations, it can be seen that the mean Doppler-shifted photon depth increases with the fibre source-detector separation and with the laser wavelength. The results obtained for skin are similar to the ones obtained by Fredriksson et al. (2009). The results obtained for the phantom are higher when compared with skin simulated results. We can explain these differences by the physical structure of the phantom that is different from the physical structure of the skin. Moreover, the phantom optical properties are different from the ones of simulated skin. Figure 10 shows a software window with the power spectrum obtained in the phantom for a wavelength of $635 \mathrm{~nm}$ and for different velocities: $7.78 \mathrm{~mm} / \mathrm{s}$ (light green); $6.25 \mathrm{~mm} / \mathrm{s}$ (red), $4.68 \mathrm{~mm} / \mathrm{s}$ (pink); $3.12 \mathrm{~mm} / \mathrm{s}$ (green) and $1.56 \mathrm{~mm} / \mathrm{s}$ (blue). The spectra were taken with a $1.2 \mathrm{~mm}$ source detector separation. As it was expected the power spectrum increases with the velocity.

The mentioned works prove the great importance of Monte Carlo simulations applied to LDF measurements.

\section{References}

Binzoni, T., Leung, T. S. \& Van De Ville, D. (2009). The photo-electric current in laserDoppler flowmetry by Monte Carlo simulations. Physics in Medicine and Biology, Vol. 54, No. 14, N303-318.

Bonner, R. \& Nossal, R. (1981). Model for laser Doppler measurements of blood flow in tissue. Applied Optics, Vol. 20, No. 12, 2097-2107.

Cal, K., Zakowiecki, D. \& Stefanovska, J. (2010). Advanced tools for in vivo skin analysis. International Journal of Dermatology, Vol. 49, No. 5, 492-499.

Clough, G., Chipperfield, A., Byrne, C., de Mul, F. \& Gush, R. (2009). Evaluation of a new high power, wide separation laser Doppler probe: potential measurement of deeper tissue blood flow. Microvascular Research, Vol. 78, No. 2, 155-161. 


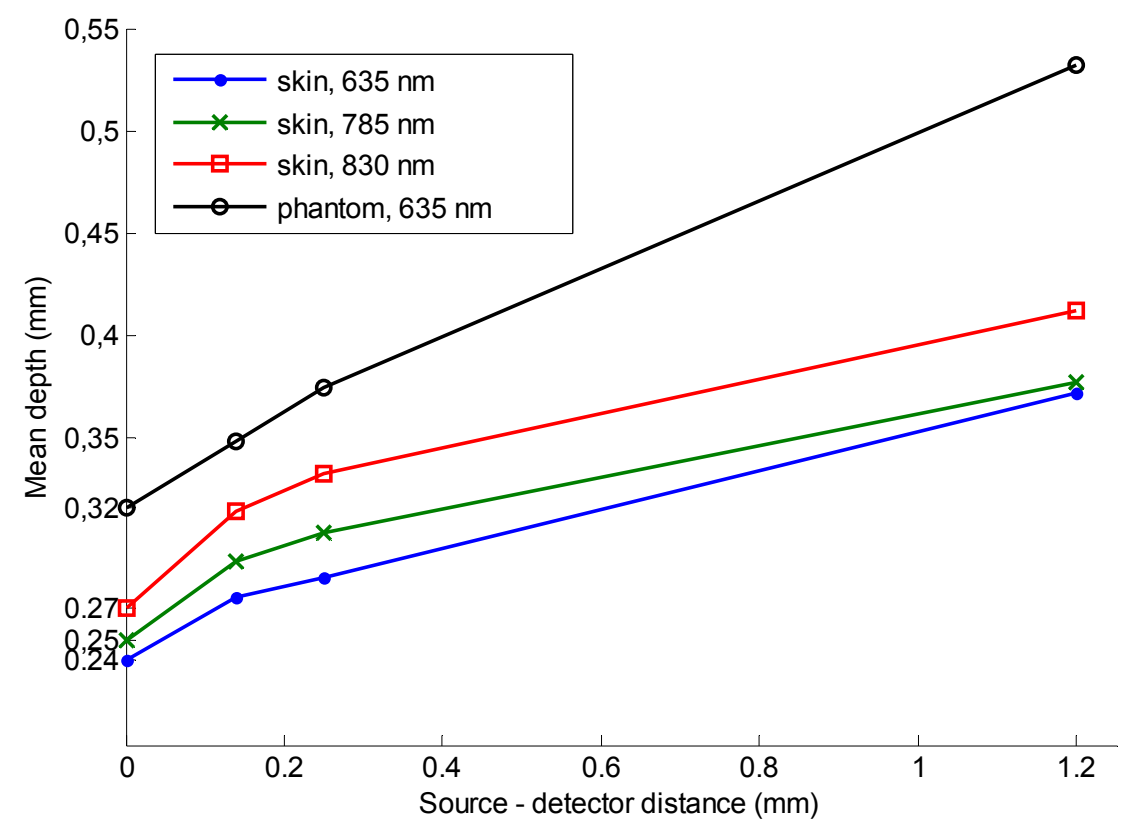

Fig. 9. Mean photon Doppler shifted depths obtained in skin simulations for different wavelengths: $635 \mathrm{~nm}$ (blue curve) with $\mu_{a}$ and $\mu_{s}$ equal to 0.15 and $20 \mathrm{~mm}^{-1}$, respectively for skin layers, and with $\mu_{a}$ and $\mu_{s}$ equal to 0.34 and $16 \mathrm{~mm}^{-1}$, respectively for blood; $785 \mathrm{~nm}$ (green curve) with $\mu_{a}$ and $\mu_{s}$ equal to 0.1 and $13 \mathrm{~mm}^{-1}$, respectively for skin layers, and with $\mu_{a}$ and $\mu_{s}$ equal to 0.5 and $13 \mathrm{~mm}^{-1}$, respectively for blood; and $830 \mathrm{~nm}$ (red curve) with $\mu_{a}$ and $\mu_{s}$ equal to 0.0122 and $18 \mathrm{~mm}^{-1}$, respectively for skin layers, and with $\mu_{a}$ and $\mu_{s}$ equal to 0.52 and $11 \mathrm{~mm}^{-1}$, respectively for blood. Phantom simulations are also shown for $635 \mathrm{~nm}$ with milk pumped at $1.56 \mathrm{~mm} / \mathrm{s}$ with $\mu_{a}$ and $\mu_{s}$ equal to 0.00052 and $52 \mathrm{~mm}^{-1}$, respectively for milk, and equal to 0.001 and $167 \mathrm{~mm}^{-1}$, respectively for the tubes. Different source-detector separations $(0,0.14,0.25$ and $1.2 \mathrm{~mm})$ are used. 


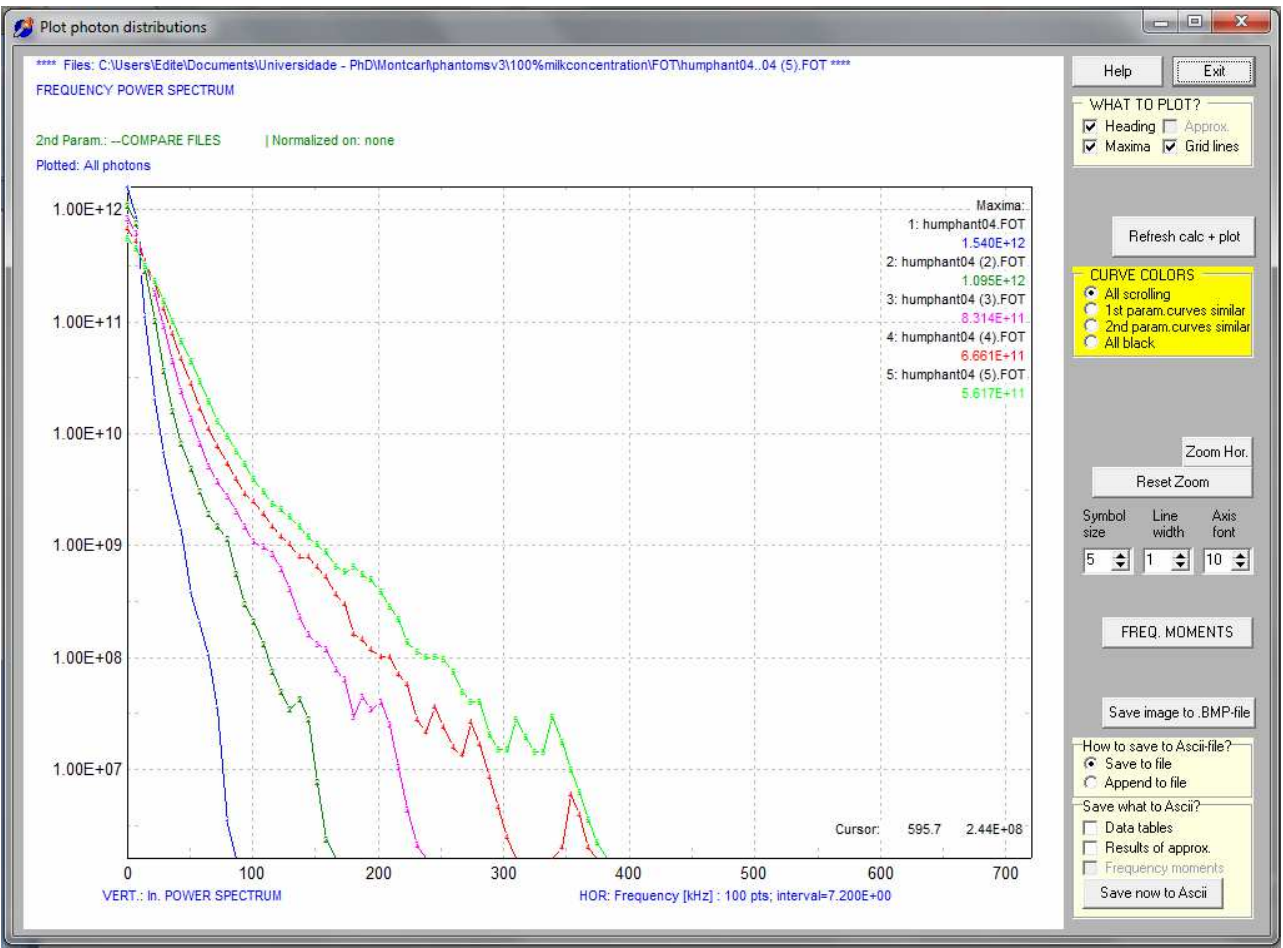

Fig. 10. Software window (software from de Mul, see www.demul.net/frits) showing the power spectrum obtained with a $635 \mathrm{~nm}$ laser source, in the phantom, for different velocities (light green: $7.78 \mathrm{~mm} / \mathrm{s}$; red: $6.25 \mathrm{~mm} / \mathrm{s}$, pink: $4.68 \mathrm{~mm} / \mathrm{s}$, green: $3.12 \mathrm{~mm} / \mathrm{s}$ and blue: 1.56 $\mathrm{mm} / \mathrm{s}$ ) and for $1.2 \mathrm{~mm}$ source-detector separation. 
de Mul, F. F. M., Koelink, M. H., Kok, M. L., Harmsma, P. J., Graaf, R. \& Aarnoudse, J. G. (1995). Laser Doppler velocimetry and Monte Carlo simulations on models for blood perfusion in tissue. Applied Optics, Vol. 34, No. 28, 6595-6611.

de Mul, F. F. M., Steenbergen, W. \& Greve, J. (1999). Doppler Monte Carlo simulations of light scattering in tissue to support laser Doppler perfusion measurements. Technology and Health Care, Vol. 7, No. 2-3, 171-183.

de Mul, F. F. M. (2004). Monte-Carlo simulations of light transport in turbid media, In: Handbook of Coherent Domain Optical Methods, Biomedical Diagnostics, Environment and Material Science, Tuchin, Valery V. (Ed.), 465-533, Kluwer Publishers, ISBN 1402075766, Dordrecht, the Netherlands.

Figueiras, E., Requicha Ferreira, L.F., Humeau, A. (2010). "Phantom validation for depth assessment in laser Doppler flowmetry technique", Proceedings of EOS - Topical Meeting on Diffractive Optics, paper 2413, Koli (Finland), 14-18 February 2010; proc. ISBN: 978-3-00-024193-2.

Fredriksson, I., Larsson, M. \& Strömberg, T. (2006). Absolute flow velocity components in laser Doppler flowmetry, Proceedings of SPIE 6094 - Optical Diagnostics and Sensing $I V$, paper 60940A, 48-59, USA, January 2006, SPIE, San Jose.

Fredriksson, I., Larsson, M. \& Strömberg, T. (2008). Optical microcirculatory skin model: assessed by Monte Carlo simulations paired with in vivo laser Doppler flowmetry. Journal of Biomedical Optics, Vol. 13, No. 1, 014015.

Fredriksson, I., Larsson, M. \& Strömberg, T. (2009). Measurement depth and volume in laser Doppler flowmetry. Microvascular Research, Vol. 78, No. 1, 4-13.

Henyey, L.G. \& Greenstein, J. L. (1941). Diffuse radiation in the galaxy. The Astrophysical Journal, Vol. 93, 70-83.

Humeau, A., Koïtka, A., Abraham, P., Saumet, J. L., \& L'Huillier, J. P. (2004). Spectral components of laser Doppler flowmetry signals recorded in healthy and type 1 diabetic subjects at rest and during a local and progressive cutaneous pressure application: scalogram analyses. Physics in Medicine and Biology, Vol. 49, No. 17, 3957-3970.

Humeau, A., Steenbergen, W., Nilsson, H. \& Strömberg, T. (2007). Laser Doppler perfusion monitoring and imaging: novel approaches. Medical and Biological Engineering and Computing, Vol. 45, No. 5, 421-435.

Jakobsson, A. \& Nilsson, G. E. (1993). Prediction of sampling depth and photon pathlength in laser Doppler flowmetry. Medical and Biological Engineering and Computing, Vol. 31, No. 3, 301-307.

Jentink, W., de Mul, F. F. M., Hermsen, R. G. A. M., Graaf, R. \& Greve, J. (1990). Monte Carlo simulations of laser Doppler blood flow measurements in tissue. Applied Optics, Vol. 29, No. 16, 2371-2381.

Koelink, M. H., de Mul, F. F. M., Greve, J., Graaf, R., Dassel, A. C. M. \& Aarnoudse, J. G. (1994). Laser Doppler blood flowmetry using two wavelengths: Monte Carlo simulations and measurements. Applied Optics, Vol. 33, No. 16, 3549-3558.

Kolinko, V. G., de Mul, F. F. M., Greve J. \& Priezzhev A. V. (1997). On refraction in MonteCarlo simulations of light transport through biological tissues. Medical and Biological Engineering and Computing, Vol. 35, No. 3, 287-288. 
Larsson, M., Steenbergen, W. \& Strömberg, T. (2002). Influence of optical properties and fiber separation on laser Doppler flowmetry. Journal of Biomedical Optics, Vol. 7, No. 2, 236-243.

Larsson, M., Nilsson, H. \& Strömberg, T. (2003). In vivo determination of local skin optical properties and photon path length by use of spatially resolved diffuse reflectance with applications in laser Doppler flowmetry. Applied Optics, Vol. 42, No. 1, 124134.

Larsson, M. \& Strömberg, T. (2006). Towards a velocity-resolved microvascular blood flow measure by decomposition of the laser Doppler spectrum. Journal of Biomedical Optics, Vol. 11, No. 1, 14024-14033.

Liebert, A., Zolek, N. \& Maniewski, R. (2006). Decomposition of a laser-Doppler spectrum for estimation of speed distribution of particles moving in an optically turbid medium: Monte Carlo validation study. Physics in Medicine and Biology, Vol. 51, No. 22, 5737-5751.

Merz, K. M., Pfau, M., Blumenstock, G., Tenenhaus, M., Schaller, H. E., Rennekampff, H. O. (2010). Cutaneous microcirculatory assessment of the burn wound is associated with depth of injury and predicts healing time. Burns, Vol. 36, No. 4, 477-482.

Nilsson, H., Larsson, M., Nilsson, G. E. \& Strömberg, T. (2002). Photon pathlength determination based on spatially resolved diffuse reflectance. Journal of Biomedical Optics, Vol. 7, No. 3, 478-485.

Öberg, P. A. (1990). Laser-Doppler flowmetry. Critical Reviews in Biomedical Engineering, Vol. 18, No. 2, 125-163.

Rajan, V., Varghese, B., van Leeuwen, T. G. \& Steenbergen, W. (2008). Influence of tissue optical properties on laser Doppler perfusion imaging, accounting for photon penetration depth and the laser speckle phenomenon. Journal of Biomedical Optics, Vol. 13, No. 2, 024001.

Rajan, V., Varghese, B., van Leeuwen, T. G. \& Steenbergen, W. (2009). Review of methodological developments in laser Doppler flowmetry. Lasers in Medical Science, Vol. 24, No. 2, 269-283.

Ray, S. A., Buckenham, T. M., Belli, A. M., Taylor, R. S. \& Dormandy, J. A. (1999). The association between laser Doppler reactive hyperaemia curves and the distribution of peripheral arterial disease. European Journal of Vascular and Endovascular Surgery, Vol. 17, No. 3, 245-248.

Smit, J. M., Zeebregts, C. J., Acosta, R. \& Werker, P. M. (2010). Advancements in free flap monitoring in the last decade: a critical review. Plastic and Reconstructive Surgery, Vol. 125, No. 1, 177-185.

Steenbergen, W. \& de Mul, F. (1997). New optical tissue phantom, and its use for studying laser Doppler blood flowmetry, Proceedings of SPIE - Optical and Imaging Techniques for Biomonitoring III, Vol. 3196, ISBN: 9780819466440, Italy, September 1997, SPIE, Italy.

Van de Hulst, H. C., Light Scattering by Small Particles, Dover Publications, New York, USA, 1957, 1981, ISBN 0-486-64228-3.

Varghese, B., Rajan, V., van Leeuwen, T. G. \& Steenbergen, W. (2007). Discrimination between Doppler-shifted and non-shifted light in coherence domain path length 
resolved measurements of multiply scattered light. Optics Express, Vol. 15, No. 20, 13340-13350.

Wang, L. and Jacques, S. L. (1993). Hybrid model of Monte-Carlo simulation and diffusion theory for light reflectance by turbid media. Journal of the Optical Society of America A, Vol. 10, No. 8, 1746-1752.

Waterworth, M. D., Tarte, B. J., Joblin, A. J., van Doorn, T. \& Niesler, H. E. (1995). Optical transmission properties of homogenized milk used as a phantom material in visible wavelength imaging. Australasian Physical and Engineering Sciences in Medicine, Vol. 18, No. 1, 39-44.

Yamamoto-Suganuma, R. \& Aso, Y. (2009). Relationship between post-occlusive forearm skin reactive hyperaemia and vascular disease in patients with Type 2 diabetes--a novel index for detecting micro- and macrovascular dysfunction using laser Doppler flowmetry. Diabetic Medicine, Vol. 26, No. 1, 83-88.

Ziegler, S., Gschwandtner, M., Zöch, C., Barth, A., Minar, E., Rüdiger, H. \& Osterode, W. (2004). Laser Doppler anemometry distinguishes primary Raynaud phenomenon from VWF syndrome. Microvascular Research, Vol. 68, No. 3, 203-208. 


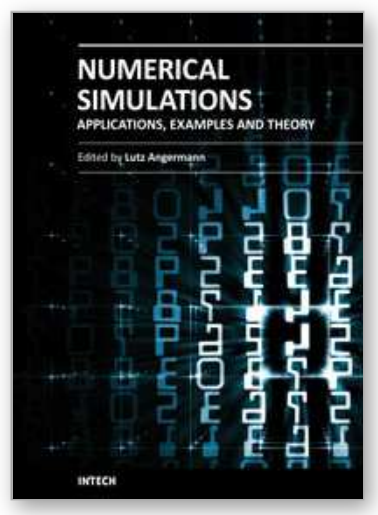

\author{
Numerical Simulations - Applications, Examples and Theory \\ Edited by Prof. Lutz Angermann
}

ISBN 978-953-307-440-5

Hard cover, 520 pages

Publisher InTech

Published online 30, January, 2011

Published in print edition January, 2011

This book will interest researchers, scientists, engineers and graduate students in many disciplines, who make use of mathematical modeling and computer simulation. Although it represents only a small sample of the research activity on numerical simulations, the book will certainly serve as a valuable tool for researchers interested in getting involved in this multidisciplinary ïneld. It will be useful to encourage further experimental and theoretical researches in the above mentioned areas of numerical simulation.

\title{
How to reference
}

In order to correctly reference this scholarly work, feel free to copy and paste the following:

Edite Figueiras, Luis F. Requicha Ferreira, Frits F.m. De Mul and Anne Humeau (2011). Monte Carlo Methods to Numerically Simulate Signals Reflecting the Microvascular Perfusion, Numerical Simulations - Applications, Examples and Theory, Prof. Lutz Angermann (Ed.), ISBN: 978-953-307-440-5, InTech, Available from: http://www.intechopen.com/books/numerical-simulations-applications-examples-and-theory/monte-carlomethods-to-numerically-simulate-signals-reflecting-the-microvascular-perfusion

\section{INTECH}

open science | open minds

\section{InTech Europe}

University Campus STeP Ri

Slavka Krautzeka 83/A

51000 Rijeka, Croatia

Phone: +385 (51) 770447

Fax: +385 (51) 686166

www.intechopen.com

\section{InTech China}

Unit 405, Office Block, Hotel Equatorial Shanghai

No.65, Yan An Road (West), Shanghai, 200040, China

中国上海市延安西路65号上海国际贵都大饭店办公楼405单元

Phone: +86-21-62489820

Fax: +86-21-62489821 
(C) 2011 The Author(s). Licensee IntechOpen. This chapter is distributed under the terms of the Creative Commons Attribution-NonCommercialShareAlike-3.0 License, which permits use, distribution and reproduction for non-commercial purposes, provided the original is properly cited and derivative works building on this content are distributed under the same license. 